\title{
Overexpression of microRNA-497 suppresses cell proliferation and induces apoptosis through targeting paired box 2 in human ovarian cancer
}

\author{
ZHONG LIN $^{1}$, JUNLING ZHAO $^{2}$, XINDAN WANG $^{3}$, XUEHONG ZHU $^{2}$ and LIANSHENG GONG ${ }^{1}$ \\ ${ }^{1}$ Hepatobiliary and Enteric Surgery Research Center, Xiangya Hospital, Central South University, Changsha, Hunan; \\ ${ }^{2}$ Department of Gynaecology, Ruikang Hospital Affiliated to Guangxi University of Chinese Medicine, Nanning, Guangxi; \\ ${ }^{3}$ Department of Gynaecology, Liuzhou Maternity and Child Healthcare Hospital, Liuzhou, Guangxi, P.R. China
}

Received February 3, 2016; Accepted March 22, 2016

DOI: $10.3892 /$ or.2016.5012

\begin{abstract}
MicroRNAs are a class of endogenous, small non-coding RNAs which are tightly involved in evolution and progression of human cancers. MicroRNA-497 has been reported as tumor-suppressor in various human cancer. However, the role of miR-497 in ovarian cancer is still poorly known. We investigated the expression level and cellular function of miR-497 in human ovarian cancer. In this study, the expression of miR-497 in ovarian cancer tissues and SKOV3 cells was detected by quantitative reverse-transcription polymerase chain reaction (qRT-PCR). CCK-8 assay was used to analysis the cell proliferation. Transwell assay was performed to analysis cell migration and invasion. Cell apoptosis was evaluated by flow cytometry. Luciferase assay was performed to verify a putative target site of miR-497 in the 3'UTR of PAX2 mRNA. The results showed that miR-497 was markedly decreased in ovarian cancer tissues and SKOV3 cells. Moreover, overexpression of miR-497 in SKOV3 cells induced PAX2 protein expression and resulted in inhibition of cell proliferation, migration and invasion, and induction of cell apoptosis. In addition, we confirmed that $P A X 2$ is a direct target gene of miR-497. Furthermore, Silencing of PAX2 by RNA interference suppressed cell proliferation and promoted cell apoptosis in vitro. Taken together, our study rationally present that miR-497 has a potential role as a useful diagnostic and therapeutic biomarker for human ovarian cancer.
\end{abstract}

Correspondence to: Professor Liansheng Gong, Department of Biliary Pancreatic Surgery, Xiangya Hospital, Central South University, 87 Xiangya Road, Changsha, Hunan 410008, P.R. China E-mail: gonglianshenglb@sina.com

Key words: ovarian cancer, miR-497, PAX2, migration and invasion, biomarker

\section{Introduction}

Ovarian carcinoma is the fifth leading cause of death among the female population, which accounts for approximate $3 \%$ of cancers among women, and it is one of the most lethal gynecologic malignancies (1). Although the strategies for cancer therapy have been developed for the last two decades, ovarian cancer mortality has not improved (1). Thus, estimating the risk of future progression and develop early diagnosis biomarker may have significant impact on ovarian carcinoma prognosis. For the last 10 years, intensive research efforts have been made toward the diagnostic and treatment of ovarian carcinoma to promote the long-term survival rate of ovarian cancer patient. However, without real improvement (2). Therefore, there is an urgent need to identify potential indicators for more effective therapies for human ovarian cancer.

MicroRNAs (miRNAs) are a class of non-coding, small endogenous RNAs with 18-22 nucleotides in length playing important roles in post-transcriptional regulation (3). miRNAs usually imperfectly bind to the 3'-untranslated region (3'UTR) of complementary target mRNA resulting in mRNA degradation or prevention translation $(4,5)$. miRNAs play important roles in various normal cellular and biological processes, such as cell proliferation, differentiation, and apoptosis (6-8). Increasing focus on the roles of miRNAs in human cancer have been reported. Previous studies have shown that 98 miRNAs locate at genomic regions involved in cancers, indicating that miRNAs are tightly interrelated with occurrence and development of cancers (9). MicroRNAs also play critical roles in carcinogenesis and progression (10), and can act as either oncogenes or tumor suppressors (11). miR-497 was first identified in neuroblastoma and acts as a predictive indicator for neuroblastoma patient survival (12). The expression of miR-497 was significantly downregulated in a variety of human cancers and acted as a tumor suppressor (13-15). However, the expression level of miR-497 and its exact role in evolution and progression of human ovarian cancer is still unclear.

In our present study, which aims to investigate the functions of dysregulated miR-497 in human ovarian cancer, we confirmed that the expression of miR-497 was significantly 
Table I. Primer sequences used for miRNA and mRNA expression analysis.

Name

Primer sequence (5'-3')

miR-497-RT
U6-RT
U6-F
U6-R
miR-497-F
Universal-R
PAX2-F
PAX2-R
GAPDH-F
GAPDH-R

CTCAACTGGTGTCGTGGAGTCGGCAATTCAGTTGAGACAAACCA CGCTTCACGAATTTGCGTGTCAT

CTCGCTTCGGCAGCACA

AACGCTTCACGAATTTGCGT

ACACTCCAGCTGGGCAGCAGCACACT

TGGTGTCGTGGAGTCG

CCTCGCTCCAATGGTGAGAA

TGCTGCTGGGTGAAGGTGTC

ACACCCACTCCTCCACCTTT

TTACTCCTTGGAGGCCATGT

F, forward; R, reverse; RT, reverse transcription.

decreased in human ovarian cancer. In addition, we also evaluated the effect of overexpression of miR-497 on human ovarian cancer cell proliferation, invasion, migration and cell apoptosis. Moreover, we further confirmed that paired box 2 (PAX2) is a directly target gene of miR-497. Our findings suggest that miR-497 acts as a tumor suppressor and may represent novel diagnostic and therapeutic biomarker for human ovarian cancer, and miR-497-PAX2 may represent a potential therapeutic target for diagnosis and therapy of ovarian cancer.

\section{Materials and methods}

Tissue collection. Between 2014 and 2015, 26 ovarian cancer tissues and para-carcinoma tissues were obtained from patients through surgery at Hepatobiliary and Enteric Surgery Research Center, Xiangya Hospital, and were diagnosed with ovarian cancer based on histopathological evaluation according to the International Federation of Obstetrics and Gynecology (FIGO) criteria. All patients were operated without any local or systemic treatment. All samples were collected and frozen in liquid nitrogen immediately, and then stored at $-80^{\circ} \mathrm{C}$ for RNA or protein extraction. All participants provided consent for the use of their specimens in this research, and the study was approved by the Institute Research Ethics Committee of Xiangya Hospital. All procedures performed in studies involving human participants were in accordance with the Ethical Standards of the Institutional and/or National Research Committee and with the 1964 Helsinki Declaration and its later amendments or comparable ethical standards.

Cell lines. Human ovarian surface immortalized epithelial cells (IOSE25) were cultured as described (16). Human ovarian cancer cell line SKOV3 was purchased from the American Type Culture Collection (ATCC; Manassas, VA, USA). The ovarian cancer cell SKOV3 was cultured according to the vendor's instructions. In brief, cells were cultured in McCoy's 5A medium modified (ATCC) with 10\% fetal bovine serum (FBS; Gibco, Carlsbad, CA, USA), and the medium contained $1 \%$ penicillin-streptomycin $(100 \mathrm{U} / \mathrm{ml}$ penicillin and $100 \mu \mathrm{g} / \mathrm{ml}$ streptomycin). All cells were cultured and maintained under standard conditions.
Plasmid construction. PAX2 3'UTR containing the putative binding sites for miR-497 was cloned from the genome DNA and constructed into downstream of psi-CHECK2 vector (Promega), named PAX2-3'UTR-WT. PAX2 mutant 3'UTR recombinant plasmid was produced by QuikChange Site-Directed Mutagenesis kit (Stratagene, USA), which generated a mutation of $7 \mathrm{bps}$ from UGCUGCU to ACGACGG in the predicted miR-497 target binding sites, named as PAX2-3'UTR-MUT. All plasmids were confirmed by DNA sequencing.

Cell transfection. The miR-497 mimics and mock were purchased from a commercial manufacturer (Ribobio, China). PAX2-siRNA (5'-GAAGUCAAGUCGAGUCUAU-3') and negative control siRNA (NC-siRNA) (5'-AGGUAGUGUAAU CGCCUUGTT-3') were obtained from Genechem Biotech (Shanghai, China). Cells $\left(1 \times 10^{4}\right)$ were seeded in a 24 -well plate and incubated for $24 \mathrm{~h}$, then ovarian cancer cells were transfected with mimics $(100 \mathrm{mM})$, siRNA $(100 \mathrm{mM})$ or vectors $(2 \mu \mathrm{g})$ using Lipofectamine 2000 (Invitrogen, Carlsbad, CA, USA) in serum-free medium in accordance with the manufacturer's instructions.

Quantitative real-time PCR ( $q R T-P C R)$ analysis. Total RNA from tissues or cultured cells was extracted using TRIzol reagent (Invitrogen Inc.). Two micrograms of total RNA was used for cDNA synthesis by using M-MLV Reverse Transcriptase (Promega, USA). The relative expression of miRNA-497 was analyzed using a SYBR PrimeScript miRNA RT-PCR kit (Takara, China) and results were normalized to U6. SYBR Green Real-Time Master Mix (Toyobo, Japan) was used to perform quantitative real-time PCR to detect the relative expression of PAX2, and GAPDH was used as an internal control. The gene-specific primers were synthesized by Sangon (Shanghai, China) (Table I). qRT-PCR and data collection were performed on Applied Biosystems 7500 Sequence detection system (ABI, USA). The miR-497 and PAX2 expression levels were calculated using the $2^{-\Delta \Delta \mathrm{Ct}}$ method.

Western blot analysis. Total proteins from tissues or cultured cells were extracted with SDS lysis buffer (Beyotime, China) 

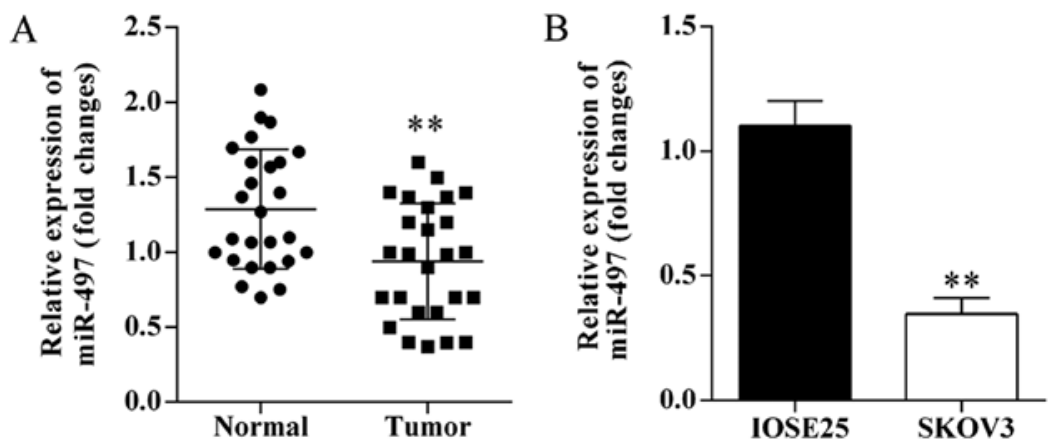

Figure 1. miR-497 is decreased in human ovarian cancer tissues and cells. (A) The expression of miR-497 was significantly decreased in human ovarian cancer tissues compared with non-tumor tissues $(\mathrm{n}=26)$. (B) miR-497 was significantly downregulated in ovarian cancer $\mathrm{SKOV} 3$ cells. ${ }^{* *} \mathrm{P}<0.01$.

and BCA protein assay kit (Pierce, USA) was used to detect the protein concentration. Equal amounts of total proteins were boiled using loading buffer for $10 \mathrm{~min}$ and separated in $12 \%$ sodium dodecyl sulfate-polyacrylamide gel electrophoresis (SDS-PAGE) and transferred to polyvinylidene difluoride membranes (PVDF) (Millipore, USA). The membranes were blocked in Tris-buffered saline (TBS) containing 5\% nonfatdried milk at $37^{\circ} \mathrm{C}$ for $2 \mathrm{~h}$ and incubated with PAX2 antibodies (1:800; Abcam) for $1 \mathrm{~h}$ at room temperature, then membranes were incubated with goat anti-rabbit IgG-HRP (1:2,000; Santa Cruz Biotechnology, USA) at $37^{\circ} \mathrm{C}$ for $1 \mathrm{~h}$. Proteins were detected by enhanced chemiluminescence (ECL; Beyotime). GAPDH (1:2,000; Abcam), a constitutive expression gene, was used as an internal control.

Dual-luciferase assays. Cells $(100 \mu \mathrm{l})\left(1 \times 10^{6} / \mathrm{ml}\right)$ were cultured in 24-well plates and incubated for $24 \mathrm{~h}$. miR-497 mimics or Mock (100 nM) were co-transfected with $20 \mathrm{ng}$ PAX2-3'UTR-WT or -MUT psi-CKECK2 vectors into cells using Lipofectamine 2000 reagent. Dual-luciferase reporter assay system (Promega) was used to detect luciferase activities after $48 \mathrm{~h}$ of transfection. Firefly luciferase activity was normalized to Renilla luciferase activity.

CCK- 8 assay. The cell proliferation was analyzed using Cell

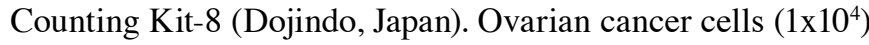
were seeded in 96-well plate. After transfected with mimics or siRNA, cells were cultured with $10 \%$ FBS for 24,48 and $72 \mathrm{~h}$. Then, the cells were treated with $10 \mu \mathrm{l}$ CCK- 8 solution at $37^{\circ} \mathrm{C}$ for $2 \mathrm{~h}$, The absorbency at $450 \mathrm{~nm}$ was measured using a microplate reader Thermo Plate (Rayto Life and Analytical Science Co., Ltd., Germany).

Cell migration and invasion assays. Transwell insert (24-well) with $8-\mu \mathrm{m}$ pore size (Corning Costar Corporation) was used to perform migration assay and invasion assay. For migration assay, $1 \times 10^{4}$ SKOV3 cells were plated in $100 \mu \mathrm{l}$ medium without FBS into upper chamber of Transwell insert with non-coated membrane. For invasion assay, $1 \times 10^{5}$ SKOV3 cells suspended in $100 \mu \mathrm{l}$ serum-free medium were loaded in the upper Matrigel-coated chamber instead. In both assays, $500 \mu \mathrm{l}$ of culture medium containing $15 \%$ FBS was added to the lower chamber for culture. Then, cells were allowed to migrate or invade for $24 \mathrm{~h}$ at $37^{\circ} \mathrm{C}$. The migrated or invaded SKOV3 cells were fixed with $100 \%$ methanol for $30 \mathrm{~min}$ at room temperature and stained with $0.5 \%$ crystal violet (Sigma, USA) for $15 \mathrm{~min}$, and the permeating cells were counted under a phase-contrast microscope (Olympus, Tokyo, Japan).

Flow cytometry. Annexin V-FITC/PI apoptosis detection kit (Sigma) was used to detect cell apoptosis by flow cytometry. In brief, after transfected with mimics or siRNA for $48 \mathrm{~h}$, $1 \times 10^{6}$ SKOV3 cells were harvested and stained using the Annexin V-FITC/PI apoptosis detection kit according to the manufacturer's instructions. Cells were analyzed using Becton-Dickinson flow cytometer. Annexin V-FITC(+)/PI(-) and Annexin V-FITC(+)/PI(+) represent the early cell apoptosis and late apoptosis/necrosis, respectively.

Statistical analysis. All results are expressed as the mean \pm SD from three independent experiments. Statistical analysis was performed using SPSS 20.0 software (SPSS Inc., USA). Statistical significance between groups was determined using a Student's t-test or one-way analysis of variance (ANOVA) test. $\mathrm{P}$-value of $<0.05$ was considered to be statistically significant.

\section{Results}

miRNA-497 is decreased in ovarian cancer tissues and cells. The expression levels of miR-497 in human ovarian cancer tissues and cells were investigated using qRT-PCR. miRNA-497 was significantly decreased in ovarian cancer tissues compared with matched adjacent normal tissues (Fig. 1A). Furthermore, the expression of miR-497 was significantly downregulated in SKOV3 cells compared to IOSE25 cells (Fig. 1B).

Overexpression of miR-497 suppresses SKOV3 cell proliferation, migration and invasion. To determine the role of miR-497 in cell proliferation, migration and invasion, SKOV3 cells were transfected with miR-497 mimics (Fig. 2A). The SKOV3 cell proliferation was significantly inhibited after transfection of miR-497 mimics (Fig. 2B). Migration and invasion abilities of SKOV3 cells after overexpression of miR-497 were analyzed using Transwell assay. The SKOV3 cell migration and invasion were significantly suppressed after transfection of miR-497 mimics (Fig. 2C and D).

Overexpression of miR-497 induces cell apoptosis. To evaluate the effect of miR-497 on SKOV3 cell apoptosis, cell apoptosis assay was performed using the Annexin V-FITC/PI staining 

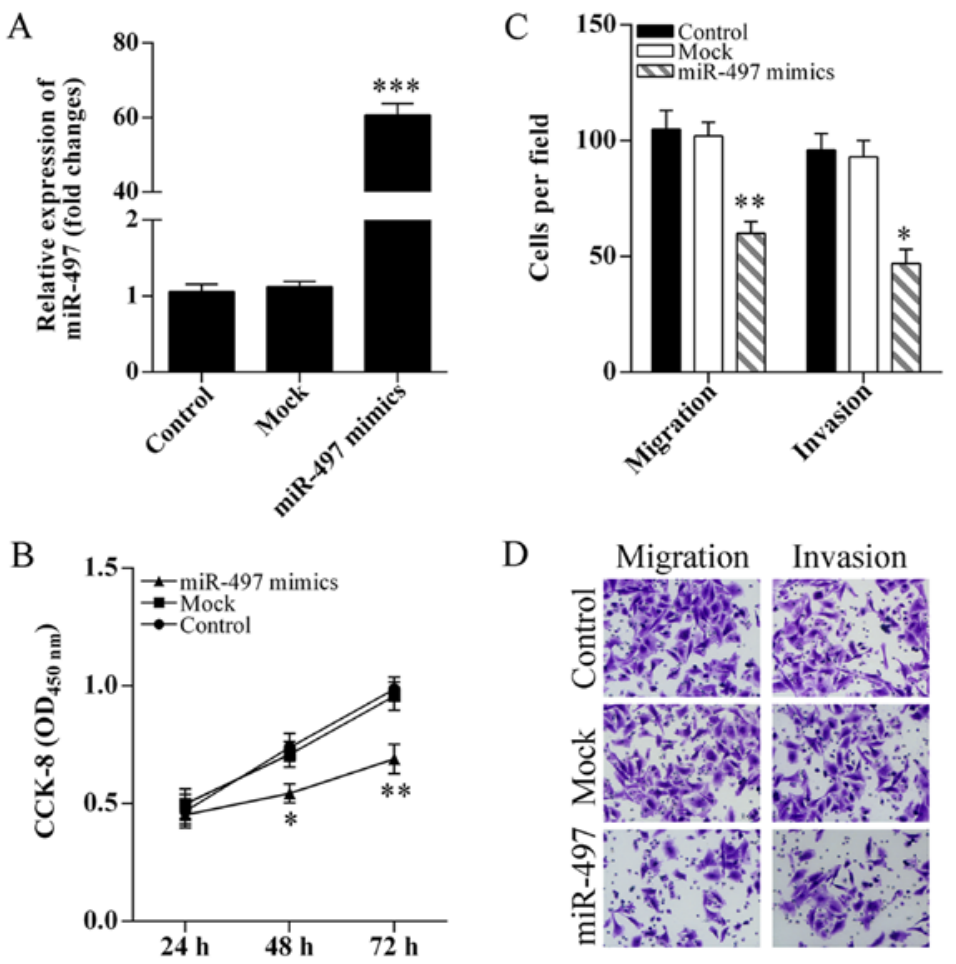

Figure 2. Overexpression of miR-497 suppresses SKOV3 cell proliferation, migration and invasion. (A) The expression of miR-497 was significantly upregulated in SKOV3 cells after transfection of miR-497 mimics. ${ }^{* * *} \mathrm{P}<0.001$. (B) CCK-8 assay results showed that overexpression of miR-497 inhibited cell proliferation. ${ }^{*} \mathrm{P}<0.05,{ }^{* *} \mathrm{P}<0.01$. (C) The average migration and invasion cell number per field among different groups. Three independent experiments were performed, ${ }^{*} \mathrm{P}<0.05,{ }^{* *} \mathrm{P}<0.01$. (D) Images of migration and invasion cells of each group ( $\mathrm{x} 40$ magnification).

A
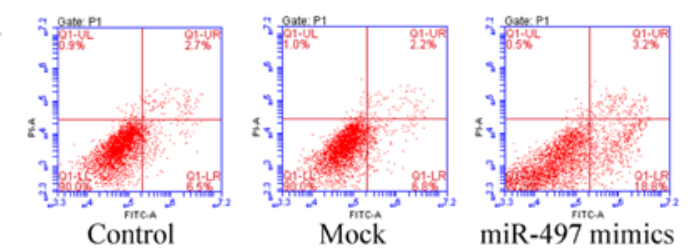

B

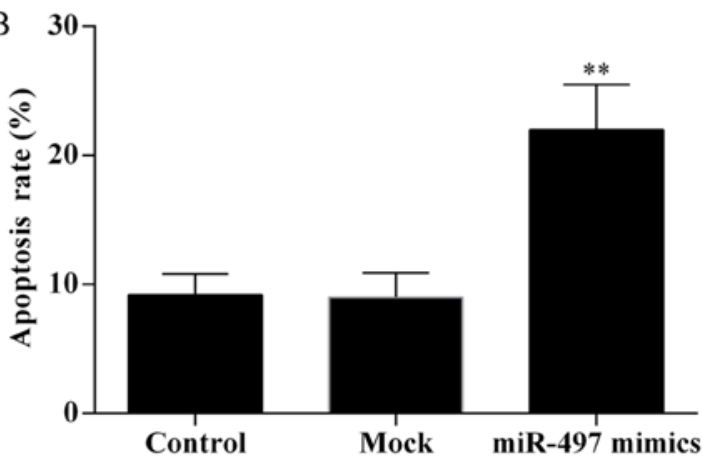

Figure 3. Overexpression of miR-497 induces SKOV3 cell apoptosis. (A) Flow cytometry analysis of SKOV3 cell apoptosis after transfection of miR-497 mimics. LL, viable cells; LR, early apoptotic cells; UR, late apoptotic cells; UL, necrotic cells. (B) The apoptosis rate of SKOV3 cells in each group. ${ }^{* *} \mathrm{P}<0.01$.

method by flow cytometry. The results showed that overexpression of miR-497 induced SKOV3 cell apoptosis (Fig. 3).

Paired box 2 (PAX2) is a target gene of miR-497. Firstly, the protein expression of PAX2 in human ovarian cancer tissues and cells was detected using western blot analysis, the result

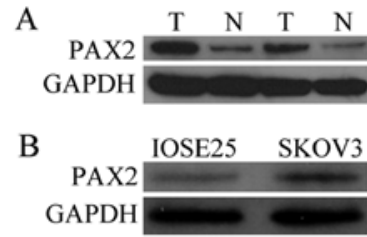

$\mathrm{C}$

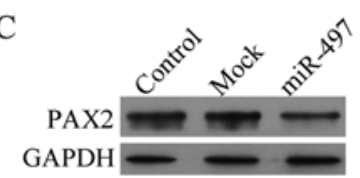

$\mathrm{D}$

\section{3' UGUUUGGUGUCACACGACGAC 5' miR-497 |||| $\mid 1$ \\ 1219: 5' AAGCCCACCAGGCUGCUGCUU 3' PAX2 WT \\ 1219: 5' AAGCCCACCAGGCACGACGGU 3' PAX2 WT}

Figure 4. PAX2 is a target gene of miR-497. (A) The protein expression of PAX2 in ovarian cancer tissues and normal tissues (T, tumor; N, normal). (B) The PAX2 protein expressed in IOSE25 and SKOV3 cells. (C) The protein expression PAX2 in SKOV3 cells after transfection of miR-497 mimics. (D) Sequence alignment of miR-497 and 3'UTR of PAX2 mRNA using TargetScan (www.targetscan.org) and MICRORNA.ORG (www.microrna. org) algorithms. (E) Luciferase activity in the PAX2-3'UTR-WT group was significantly decreased after co-transfection with miR-497 mimics. ${ }^{* *} \mathrm{P}<0.01$.

showed PAX2 was increased in tumor tissues (Fig. 4A) and SKOV3 cells (Fig. 4B). In addition, to determine that miR-497 regulates PAX2 expression directly in ovarian cancer, the expression of PAX2 in miR-497 mimic-transfected SKOV3 cells was analyzed using western blot analysis (Fig. 4C). Furthermore, miRNA target predication databases 

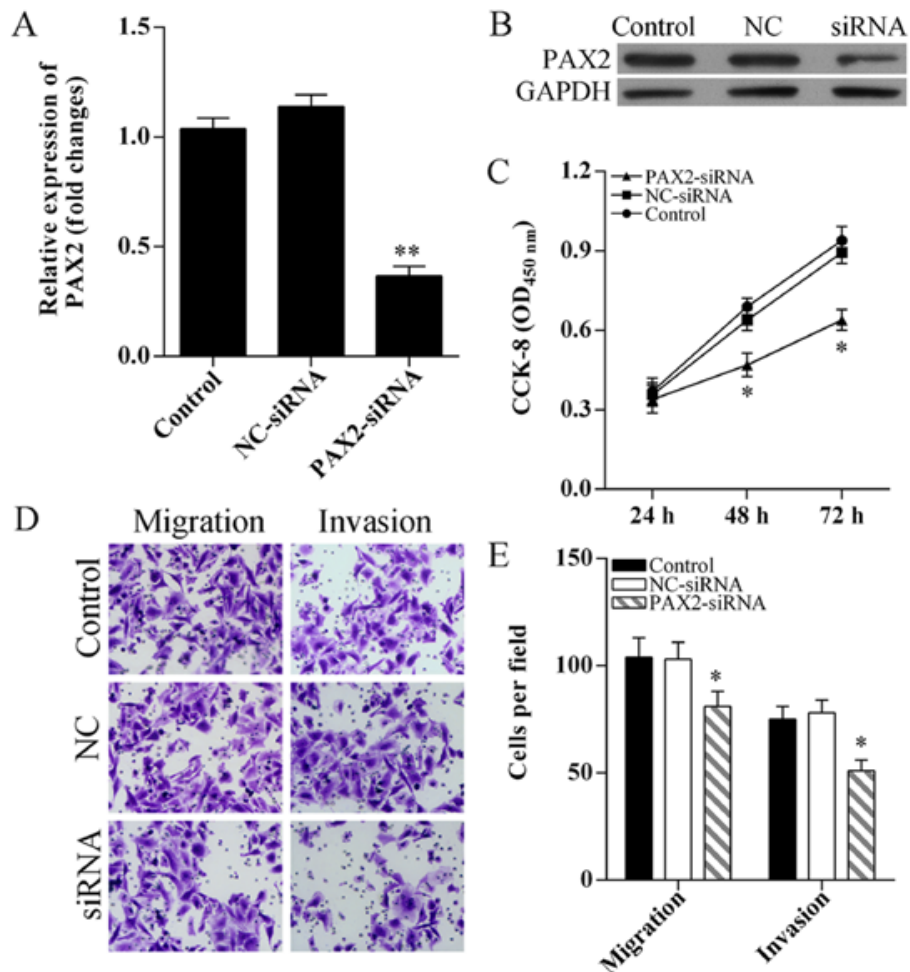

Figure 5. Knockdown of PAX2 suppresses SKOV3 cell proliferation, migration and invasion. (A) The expression of miR-497 was significantly decreased after PAX2-siRNA transfection. ${ }^{* *} \mathrm{P}<0.01$. (B) The protein expression of miR-497 in SKOV3 cells after PAX2-siRNA transfection. (C) CCK-8 assay results showed that knockdown of PAX2 inhibited cell proliferation. "P $<0.05$. (D) Images of migration and invasion cells of each group ( $\mathrm{x} 40$ magnification). (E) The average migration and invasion cell number per field among different groups. Three independent experiments were performed, ${ }^{*} \mathrm{P}<0.05$.

A
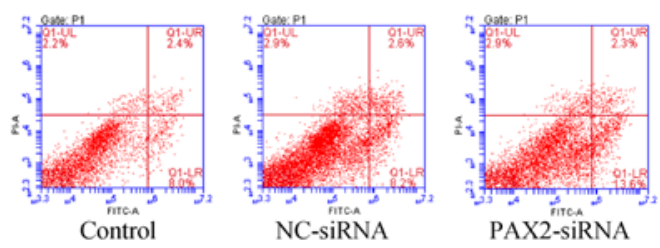

B

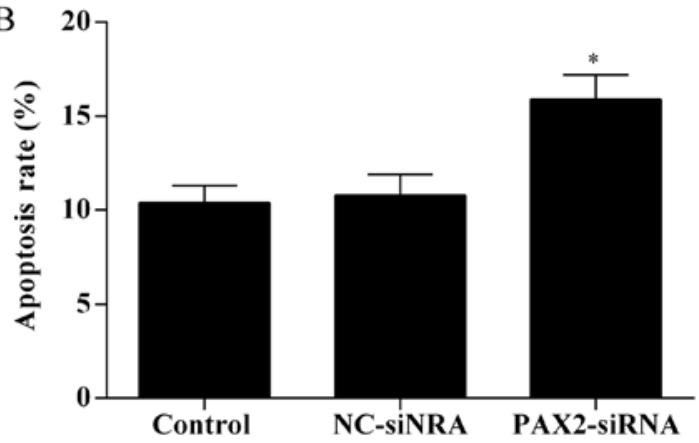

Figure 6. Knockdown of PAX2 induces SKOV3 cell apoptosis. (A) Flow cytometry analysis of SKOV3 cell apoptosis after knockdown of PAX2. LL, viable cells; LR, early apoptotic cells; UR, late apoptotic cells; UL, necrotic cells. (B) The apoptosis rate of SKOV 3 cells in each group, . " $\mathrm{P}<0.05$.

(TargetScan: www.targetscan.org and MICRORNA.ORG: www.microrna.org) were used for computational analyses. miR-497 has one predictive target site in the human PAX2 3'UTR (Fig. 4D). Moreover, dual-luciferase assay was performed to verify whether PAX2 is a direct target gene of miR-497. The results showed that miR-497 mimics suppressed the luciferase activity of the reporter (Fig. 4E). Our results indicated that this site of PAX2 3'UTR was the exact regulation site for miR-497.

Knockdown of PAX2 inhibits SKOV3 cell proliferation, migration and invasion. To determine the role of PAX2 in cell proliferation, migration and invasion, $\mathrm{PAX} 2$ was knocked down using small interference RNA (Fig. 5A and B). CCK-8 assay results showed SKOV3 cell proliferation was significantly suppressed after PAX2-siRNA transfection (Fig. 5C). Transwell assay was performed to analyzed cell migration and invasion, the results showed knockdown of PAX2 inhibited SKOV3 cell migration and invasion (Fig. 5D and E).

Knockdown of PAX2 induces SKOV3 cell apoptosis. To investigate the effect of PAX2 on ovarian cancer cell apoptosis, the Annexin V-FITC/PI staining method was used to perform apoptosis assays by flow cytometry. The results showed that knockdown of PAX2 promoted SKOV3 cell apoptosis (Fig. 6).

\section{Discussion}

In recent years, increased number of studies have reported that miRNA regulates gene expression involved in variety of biological processes including cell growth, differentiation, migration and invasion, and tumorigenesis $(4,17)$. Many miRNAs are aberrantly expressed in human cancers and unconventional expression of miRNAs result in uncontrolled tumor growth $(18,19)$. Therefore, increased understanding of how miRNA regulates cell proliferation and survival may 
provide better strategies for the diagnosis and treatment of human cancer, including ovarian cancer.

MicroRNA-497 is located on chromosome 17p13.120 and is lost in $93 \%$ of human small-cell lung cancers (21). Previous studies indicated that miR-497 acts as a tumor suppressor in human cancers. The expression of miR-497 has been found decreased in colorectal cancer (22), breast cancer (23), pancreatic cancer (24), human osteosarcoma (25), cervical cancer (26) and human lung cancer (27). In addition, downregulation of miR-497 was associated with malignancy of colorectal cancer (22) and breast cancer (23). Furthermore, overexpression of miR-497 can inhibit pancreatic cancer and breast cancer cell growth both in vitro and in vivo $(15,23)$. In our present study, the expression level of miR-497 in human ovarian cancer tissues and cells was confirmed and the role of miR-497 in ovarian cancer cell proliferation and apoptosis was evaluated. Our results showed that miR-497 was significantly decreased in human ovarian cancer tissues and cells. In addition, overexpression of miR-497 inhibited SKOV3 cell proliferation, suppressed cell migration and invasion, and promoted cell apoptosis. Furthermore, overexpression of miR-497 by mimic-transfection reduced the PAX2 protein expression, and dual-luciferase assay confirmed that the PAX2 is a target gene of miR-497. Moreover, knockdown of PAX2 by RNA interference suppressed SKOV3 cell growth, inhibited cell migration and invasion, and induced cell apoptosis. All the above indicated that miR-497 acts as a tumor suppressor by regulating cell proliferation, metastasis and apoptosis in human ovarian cancer, and miR-497-PAX2 may represent a new potential diagnosis and therapeutic target for the treatment of human ovarian cancer.

Paired box $(P A X)$ genes are named for the conserved DNA sequence motif which comprises a 128 -amino acid domain and encodes a group of transcription factors (28). Nine $P A X$ genes have been identified in mammals (29). $P A X 2$, one of the $P A X$ genes, is a critical regulator of embryogenesis (30-32). Embryonic $P A X 2$ gene expression is largely attenuated in adult tissue, such as breast (33). Loss of $P A X 2$ is associated with kidney hypoplasia and vesicoureteral reflux (34). On the contrary, upregulation of PAX2 is associated with human cancers $(35,36)$. PAX2 was significantly increased in human prostate cancer (37) and breast cancer (38). Inhibit the expression of PAX2 in renal cell carcinoma cells (39) and knock-down of PAX2 in bladder cancer cells (40) suppressed the cell growth and induced cell apoptosis. Similarly, silencing of PAX2 led to Kaposi sarcoma cell death and inhibited cell invasion and migration (41). However, the function of PAX2 in the evolution and progression of ovarian cancer is still unclear. In our present study, PAX2 was increased in human ovarian cancer tissues and cells. Silencing of PAX2 markedly suppressed SKOV3 cell proliferation, blocked cell migration and invasion, and induced cell apoptosis. We confirmed that the expression of PAX2 was regulated by miR-497, at least partly. Taken together, our findings indicate that decreasing of miR-497 may promote the proliferation capacity of human ovarian cancer through the PAX2-mediated signal pathway.

In conclusion, we demonstrated that miR-497 is decreased in human ovarian cancer tissues and cells, and acts as a tumor suppressor. We also confirmed PAX2 is a target gene of miR-497. Overexpression of miR-497 or silencing of
PAX2 suppressed the proliferative and induced apoptosis of ovarian cancer cells. Based on the above, our study present that miR-497 has the potential role as a useful diagnostic and therapeutic biomarker for human ovarian cancer.

\section{References}

1. Torre LA, Bray F, Siegel RL, Ferlay J, Lortet-Tieulent J and Jemal A: Global cancer statistics, 2012. CA Cancer J Clin 65: 87-108, 2015

2. Breuer EK and Murph MM: The role of proteomics in the diagnosis and treatment of women's cancers: Current trends in technology and future opportunities. Int J Proteomics 2011: pii: 373584, 2011.

3. Doench JG and Sharp PA: Specificity of microRNA target selection in translational repression. Genes Dev 18: 504-511, 2004.

4. Bartel DP: MicroRNAs: Genomics, biogenesis, mechanism, and function. Cell 116: 281-297, 2004.

5. Filipowicz W, Bhattacharyya SN and Sonenberg N: Mechanisms of post-transcriptional regulation by microRNAs: Are the answers in sight? Nat Rev Genet 9: 102-114, 2008.

6. Meyer SU, Thirion C, Polesskaya A, Bauersachs S, Kaiser S, Krause S and Pfaffl MW: TNF- $\alpha$ and IGF1 modify the microRNA signature in skeletal muscle cell differentiation. Cell Commun Signal 13: 4, 2015.

7. Tian L, Fang YX, Xue JL and Chen JZ: Four microRNAs promote prostate cell proliferation with regulation of PTEN and its downstream signals in vitro. PLoS One 8: e75885, 2013.

8. Ambros V: MicroRNA pathways in flies and worms: Growth, death, fat, stress, and timing. Cell 113: 673-676, 2003.

9. Calin GA, Sevignani C, Dumitru CD, Hyslop T, Noch E, Yendamuri S, Shimizu M, Rattan S, Bullrich F, Negrini M, et al: Human microRNA genes are frequently located at fragile sites and genomic regions involved in cancers. Proc Natl Acad Sci USA 101: 2999-3004, 2004.

10. Farazi TA, Hoell JI, Morozov P and Tuschl T: MicroRNAs in human cancer. Adv Exp Med Biol 774: 1-20, 2013.

11. Lages E, Ipas H, Guttin A, Nesr H, Berger F and Issartel JP: MicroRNAs: Molecular features and role in cancer. Front Biosci (Landmark Ed) 17: 2508-2540, 2012.

12. Bray I, Bryan K, Prenter S, Buckley PG, Foley NH, Murphy DM, Alcock L, Mestdagh P, Vandesompele J, Speleman F, et al: Widespread dysregulation of miRNAs by MYCN amplification and chromosomal imbalances in neuroblastoma: Association of miRNA expression with survival. PLoS One 4: e7850, 2009.

13. Zhao X, Zhao Z, Xu W, Hou J and Du X: Down-regulation of $\mathrm{miR}-497$ is associated with poor prognosis in renal cancer. Int $\mathbf{J}$ Clin Exp Pathol 8: 758-764, 2015.

14. Itesako T, Seki N, Yoshino H, Chiyomaru T, Yamasaki T, Hidaka H, Yonezawa T, Nohata N, Kinoshita T, Nakagawa M, et al: The microRNA expression signature of bladder cancer by deep sequencing: The functional significance of the miR-195/497 cluster. PLoS One 9: e84311, 2014.

15. Xu J, Wang T, Cao Z, Huang H, Li J, Liu W, Liu S, You L, Zhou L, Zhang T, et al: miR-497 downregulation contributes to the malignancy of pancreatic cancer and associates with a poor prognosis. Oncotarget 5: 6983-6993, 2014.

16. Li NF, Broad S, Lu YJ, Yang JS, Watson R, Hagemann T, Wilbanks G, Jacobs I, Balkwill F, Dafou D, et al: Human ovarian surface epithelial cells immortalized with hTERT maintain functional $\mathrm{pRb}$ and $\mathrm{p} 53$ expression. Cell Prolif 40: 780-794, 2007

17. Lagos-Quintana M, Rauhut R, Lendeckel $\mathrm{W}$ and Tuschl T: Identification of novel genes coding for small expressed RNAs. Science 294: 853-858, 2001.

18. Liu H, Li W, Chen C, Pei Y and Long X: miR-335 acts as a potential tumor suppressor miRNA via downregulating ROCK1 expression in hepatocellular carcinoma. Tumour Biol 36: 6313-6319, 2015.

19. Tu Y, Liu L, Zhao D, Liu Y, Ma X, Fan Y, Wan L, Huang T, Cheng $Z$ and Shen B: Overexpression of miRNA-497 inhibits tumor angiogenesis by targeting VEGFR2. Sci Rep 5: 13827, 2015.

20. Bailey-Wilson JE, Amos CI, Pinney SM, Petersen GM, de Andrade M, Wiest JS, Fain P, Schwartz AG, You M, Franklin W, et al: A major lung cancer susceptibility locus maps to chromosome 6q23-25. Am J Hum Genet 75: 460-474, 2004. 
21. Girard L, Zöchbauer-Müller S, Virmani AK, Gazdar AF and Minna JD: Genome-wide allelotyping of lung cancer identifies new regions of allelic loss, differences between small cell lung cancer and non-small cell lung cancer, and loci clustering. Cancer Res 60: 4894-4906, 2000.

22. Guo ST, Jiang CC, Wang GP, Li YP, Wang CY, Guo XY, Yang RH, Feng Y, Wang FH, Tseng HY, et al: MicroRNA-497 targets insulin-like growth factor 1 receptor and has a tumour suppressive role in human colorectal cancer. Oncogene 32: 1910-1920, 2013.

23. Li D, Zhao Y, Liu C, Chen X, Qi Y, Jiang Y, Zou C, Zhang X, Liu S, Wang X, et al: Analysis of miR-195 and miR-497 expression, regulation and role in breast cancer. Clin Cancer Res 17: 1722-1730, 2011.

24. Xu JW, Wang TX, You L, Zheng LF, Shu H, Zhang TP and Zhao YP: Insulin-like growth factor 1 receptor (IGF-1R) as a target of miR-497 and plasma IGF-1R levels associated with TNM stage of pancreatic cancer. PLoS One 9: e92847, 2014.

25. Ruan WD, Wang P, Feng S, Xue Y and Zhang B: MicroRNA-497 inhibits cell proliferation, migration, and invasion by targeting AMOT in human osteosarcoma cells. Onco Targets Ther 9: 303-313, 2016

26. Luo M, Shen D, Zhou X, Chen X and Wang W: MicroRNA-497 is a potential prognostic marker in human cervical cancer and functions as a tumor suppressor by targeting the insulin-like growth factor 1 receptor. Surgery 153: 836-847, 2013.

27. Xu S, Fu G-B, Tao Z, OuYang J, Kong F, Jiang BH, Wan X and Chen K: miR-497 decreases cisplatin resistance in ovarian cancer cells by targeting mTOR/P70S6K1. Oncotarget 6: 26457-26471, 2015.

28. Eccles MR, He S, Legge M, Kumar R, Fox J, Zhou C, French M and Tsai RW: PAX genes in development and disease: The role of PAX2 in urogenital tract development. Int J Dev Biol 46 : 535-544, 2002

29. Robson EJ, He SJ and Eccles MR: A PANorama of PAX genes in cancer and development. Nat Rev Cancer 6: 52-62, 2006.

30. Luu VD, Boysen G, Struckmann K, Casagrande S, von Teichman A, Wild PJ, Sulser T, Schraml P and Moch H: Loss of VHL and hypoxia provokes PAX2 up-regulation in clear cell renal cell carcinoma. Clin Cancer Res 15: 3297-3304, 2009.

31. Chan-Ling T, Chu Y, Baxter L, Weible Ii M and Hughes S: In vivo characterization of astrocyte precursor cells (APCs) and astrocytes in developing rat retinae: Differentiation, proliferation, and apoptosis. Glia 57: 39-53, 2009.
32. Sims-Lucas S, Cusack B, Baust J, Eswarakumar VP, Masatoshi H, Takeuchi A and Bates CM: Fgfr1 and the IIIc isoform of Fgfr2 play critical roles in the metanephric mesenchyme mediating early inductive events in kidney development. Dev Dyn 240: 240-249, 2011.

33. Li CG and Eccles MR: PAX genes in cancer; Friends or foes? Front Genet 3: 6, 2012.

34. Weber S, Taylor JC, Winyard P, Baker KF, Sullivan-Brown J, Schild R, Knüppel T, Zurowska AM, Caldas-Alfonso A, Litwin M, et al: SIX2 and BMP4 mutations associate with anomalous kidney development. J Am Soc Nephrol 19: 891-903, 2008.

35. Tong GX, Chiriboga L, Hamele-Bena D and Borczuk AC: Expression of PAX2 in papillary serous carcinoma of the ovary: immunohistochemical evidence of fallopian tube or secondary Müllerian system origin? Mod Pathol 20: 856-863, 2007.

36. Tung CS, Mok SC, Tsang YT, Zu Z, Song H, Liu J, Deavers MT, Malpica A, Wolf JK, Lu KH, et al: PAX2 expression in low malignant potential ovarian tumors and low-grade ovarian serous carcinomas. Mod Pathol 22: 1243-1250, 2009.

37. Khoubehi B, Kessling AM, Adshead JM, Smith GL, Smith RD and Ogden CW: Expression of the developmental and oncogenic PAX2 gene in human prostate cancer. J Urol 165: 2115-2120, 2001.

38. Silberstein GB, Dressler GR and Van Horn K: Expression of the PAX2 oncogene in human breast cancer and its role in progesterone-dependent mammary growth. Oncogene 21: 1009$1016,2002$.

39. Gnarra JR and Dressler GR: Expression of Pax-2 in human renal cell carcinoma and growth inhibition by antisense oligonucleotides. Cancer Res 55: 4092-4098, 1995.

40. Muratovska A, Zhou C, He S, Goodyer P and Eccles MR: Paired-box genes are frequently expressed in cancer and often required for cancer cell survival. Oncogene 22: 7989-7997, 2003.

41. Buttiglieri S, Deregibus MC, Bravo S, Cassoni P, Chiarle R, Bussolati B and Camussi G: Role of Pax 2 in apoptosis resistance and proinvasive phenotype of Kaposi's sarcoma cells. J Biol Chem 279: 4136-4143, 2004. 